\title{
How does external feedback cause AIGaAs-based diode lasers to degrade?
}

\author{
Hempel, Martin; Chi, Mingjun; Petersen, Paul Michael; Zeimer, Ute; Tomm, Jens W.
}

Published in:

Applied Physics Letters

Link to article, DOI:

$10.1063 / 1.4775681$

Publication date:

2013

Document Version

Publisher's PDF, also known as Version of record

Link back to DTU Orbit

Citation (APA):

Hempel, M., Chi, M., Petersen, P. M., Zeimer, U., \& Tomm, J. W. (2013). How does external feedback cause AlGaAs-based diode lasers to degrade? Applied Physics Letters, 102(2), 023502.

https://doi.org/10.1063/1.4775681

\section{General rights}

Copyright and moral rights for the publications made accessible in the public portal are retained by the authors and/or other copyright owners and it is a condition of accessing publications that users recognise and abide by the legal requirements associated with these rights.

- Users may download and print one copy of any publication from the public portal for the purpose of private study or research.

- You may not further distribute the material or use it for any profit-making activity or commercial gain

- You may freely distribute the URL identifying the publication in the public portal 


\section{AIP Appiled Physics Letters}

\section{How does external feedback cause AIGaAs-based diode lasers to degrade?}

Martin Hempel, Mingjun Chi, Paul M. Petersen, Ute Zeimer, and Jens W. Tomm

Citation: Appl. Phys. Lett. 102, 023502 (2013); doi: 10.1063/1.4775681

View online: http://dx.doi.org/10.1063/1.4775681

View Table of Contents: http://apl.aip.org/resource/1/APPLAB/v102/i2

Published by the American Institute of Physics.

\section{Related Articles}

Rate equation analysis of injection-locked quantum cascade lasers

J. Appl. Phys. 113, 063104 (2013)

A quantum cascade laser absorption spectrometer devoted to the in situ measurement of atmospheric $\mathrm{N} 2 \mathrm{O}$ and $\mathrm{CH} 4$ emission fluxes

Rev. Sci. Instrum. 84, 023103 (2013)

High beta lasing in micropillar cavities with adiabatic layer design

Appl. Phys. Lett. 102, 052114 (2013)

Portable optical-resolution photoacoustic microscopy with a pulsed laser diode excitation

Appl. Phys. Lett. 102, 053704 (2013)

Electro-optical and lasing properties of hybrid quantum dot/quantum well material system for reconfigurable photonic devices

Appl. Phys. Lett. 102, 053110 (2013)

\section{Additional information on Appl. Phys. Lett.}

Journal Homepage: http://apl.aip.org/

Journal Information: http://apl.aip.org/about/about_the_journal

Top downloads: http://apl.aip.org/features/most_downloaded

Information for Authors: http://apl.aip.org/authors

\section{ADVERTISEMENT}

\section{AIP Applied Physics Letters}

\section{EXPLORE WHAT'S NEW IN APL}

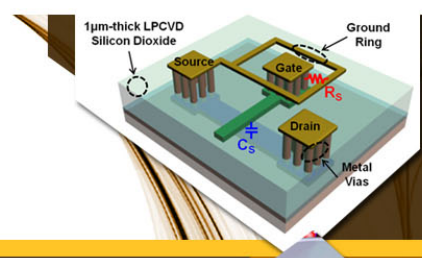

SURFACES AND INTERFACES

Focusing on physical, chemical, biological structural, optical, magnetic and electrical properties of surfaces and interfaces, and more.. 


\title{
How does external feedback cause AIGaAs-based diode lasers to degrade?
}

\author{
Martin Hempel, ${ }^{1, a)}$ Mingjun Chi, ${ }^{2}$ Paul M. Petersen, ${ }^{2}$ Ute Zeimer, ${ }^{3}$ and Jens W. Tomm ${ }^{1}$ \\ ${ }^{1}$ Max-Born-Institut, Max-Born-Str. 2 A, 12489 Berlin, Germany \\ ${ }^{2}$ DTU Fotonik, Department of Photonics Engineering, Technical University of Denmark, Frederiksborgvej \\ 399, P.O. Box 49, DK-4000 Roskilde, Denmark \\ ${ }^{3}$ Ferdinand-Braun-Institut, Leibniz-Institut für Höchstfrequenztechnik, Gustav-Kirchhoff-Str. 4, 12489 Berlin, \\ Germany
}

(Received 3 July 2012; accepted 26 December 2012; published online 15 January 2013)

\begin{abstract}
The effect of external feedback on the degradation of $808 \mathrm{~nm}$ emitting AlGaAs-based high-power broad-area diode lasers is studied. For this purpose, early stages of gradual degradation are induced by accelerated aging at high power levels. While the quantum well that actually experiences the highest total optical load remains unaffected, severe impact by point defects is observed on the cladding layers and the waveguide. Extended defects such as dislocations, however, are not observed in such early stages of degradation, which are accompanied by gradual power loss of a few percent only. (C) 2013 American Institute of Physics. [http://dx.doi.org/10.1063/1.4775681]
\end{abstract}

Operating diode lasers frequently experience external feedback $;{ }^{1}$ i.e., a fraction of their emission power is returned into the cavity containing the gain medium, which is nowadays typically a quantum well (QW). In some cases, this optical feedback is desired, e.g., if external mirrors, gratings, or fiber Bragg gratings form an "extended cavity" together with the actual diode laser chip. Such arrangements are used, for example, for stabilization or controlled tuning of the emission wavelength. Optical feedback can also be used for improving spatial and temporal coherence of broad-area diode lasers. ${ }^{2,3}$ In other cases, there might be unintentional optical feedback by reflections at optical elements, such as filters, mirrors, or lenses.

Both kinds of feedback increase the load on the diode laser. Therefore, external feedback is a possible source for degradation of diode lasers. In particular, when aligning setups with diode lasers (and in doing so modifying the amount of feedback in an uncontrolled way), sometimes sudden device degradation is observed. The appearance of the resulting damage pattern is very similar to that observed in catastrophic optical (mirror) damage $(\mathrm{CO}(\mathrm{M}) \mathrm{D}) .^{4}$

In order to make sudden degradation less likely or even to avoid it, it is highly desirable to learn about the mechanisms, by which external feedback can trigger what eventually leads to $\mathrm{CO}(\mathrm{M}) \mathrm{D}$. This represents the main goal of the present study. It relies on analysis of effects caused by gradual degradation in a diode laser that is subjected to accelerated aging under optical feedback. It appears that the cladding and waveguide layers of the diode laser structures are noticeably affected, while the QW, i.e., the actual gain medium, which doubtlessly experiences the highest total optical load, seems to be unaffected.

$808 \mathrm{~nm}$ emitting broad-area devices are investigated. ${ }^{5}$ The structure as depicted in Fig. 1(a) is grown by metal organic chemical vapor deposition. It consists of a $100 \mathrm{~nm}$ GaAs buffer, a $10 \mathrm{~nm}$ thick InAlGaAs single QW, an $\mathrm{Al}_{0.35} \mathrm{Ga}_{0.65} \mathrm{As}$-waveguide of $1 \mu \mathrm{m}$ total thickness, and about $1.2 \mu \mathrm{m}$ thick $\mathrm{Al}_{0.45} \mathrm{Ga}_{0.55} \mathrm{As}$ claddings on both sides of

\footnotetext{
${ }^{\text {a) }}$ Author to whom correspondence should be addressed. Electronic mail: tomm@mbi-berlin.de.
}

the waveguide. The laser chips (length $\times$ width $=1200$ $\times 400 \mu \mathrm{m}^{2}$ ) are packaged "p-side-down." The facets are in situ cleaned and coated with standard dielectric mirrors. The stripe is $200 \mu \mathrm{m}$ in width and again subdivided into 20 emitter stripes formed by ion-implantation. More details about these devices with a maximum emission power of $2 \mathrm{~W}$ are given elsewhere. ${ }^{6}$ A total of 8 nominally "identical" devices have been investigated. Threshold current $(560 \mathrm{~mA})$ and slope efficiency $(1.1 \mathrm{~W} / \mathrm{A})$ variation were below $1 \%$. Here, we limit the presentation to 3 devices, called P (Pristine), R (Reference, aged without feedback), and F (aged with Feedback).

Figure 1(b) depicts the setup used for the aging tests under on-axis optical feedback. A beam splitter with a reflectivity of $7.8 \%$ separates the output into a "feedback beam" and a "diagnostic beam." Optical feedback is implemented by a transmitting mirror onto which the spot is focused by lens optics to $40 \times 240 \mu \mathrm{m}^{2}$ (fast axis $\times$ slow axis). The reflectivity of $25 \%$ has been chosen to account for moderate feedback simulating practical situations. The emission in the "diagnostic beam" is either focused to a beam scanner or a power meter. The same setup is used for monitoring the aging behavior of diode lasers without feedback (e.g., device R) by blocking the "feedback beam." Devices were operated on heatsinks stabilized to $25^{\circ} \mathrm{C}$.

Figure 1(c) quantifies the degradation behavior of devices $\mathrm{R}$ and $\mathrm{F}$ during the first $200 \mathrm{~h}$ of accelerated aging with an operation current of $3 \mathrm{~A}$ (typical operation value: $2 \mathrm{~A}$ ). While without feedback, both devices show almost the same initial emission power of $(2.84 \pm 0.02) \mathrm{W}$, the external feedback increases this value for device $\mathrm{F}$ to $(2.91 \pm 0.02) \mathrm{W}$. The observed power degradation rate $(\mathrm{d} P / \mathrm{d} t)$ for device $\mathrm{R}$ (open squares) amounts to $-(0.34 \pm 0.01) \mathrm{mWh}^{-1}$, while under feedback (see, device $\mathrm{F}$, full circles), a more than doubled rate of $-(0.79 \pm 0.01) \mathrm{mWh}^{-1}$ is observed. The evolution of the output power of device $\mathrm{F}$ has also been tested without feedback. For this purpose, for each measurement, the feedback beam is blocked for a few minutes to allow the device to reach stable operation conditions. The degradation rate is $-(0.76 \pm 0.01) \mathrm{mWh}^{-1}$. This is almost 


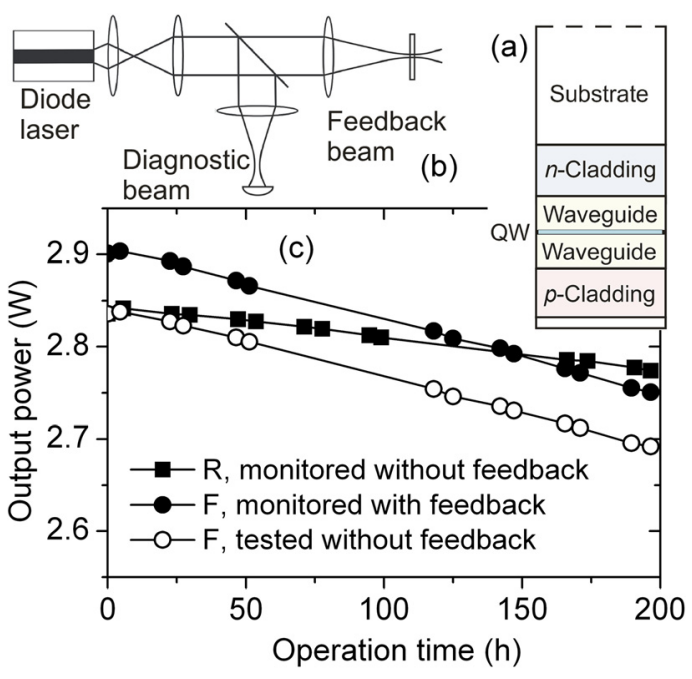

FIG. 1. (a) Schematic diagram of the epitaxial layer sequence of the devices investigated. (b) Setup for device aging under on-axis external optical feedback. (c) Power evolution for constant current aging at $3 \mathrm{~A}$ and $25^{\circ} \mathrm{C}$.

the same value as observed during monitoring the aging with external feedback. This means that the decrease of the output power for device $\mathrm{F}$ with external feedback is exclusively caused by the degradation of the device and not by any change of the external cavity (e.g., mechanical instability of the cavity) in the measuring process. The almost linear power losses of $2.4 \%$ (device $\mathrm{R}$ ) and $5.4 \%$ (device F) within the first $200 \mathrm{~h}$ of operation point to genuine gradual degradation. This is consistent with the results of careful optical inspection, which did not reveal any local external degradation signatures. Measurements of the nearfield pattern show that external feedback influences them in a reproducible way, while aging only slightly reduces the amplitude without any re-arrangement of lateral modes. The latter is consistent with the gradual character of the observed aging process.

In Figure 2, the results from monitoring the spontaneous emission from the facet of device $\mathrm{F}$ show how the $200 \mathrm{~h}$ of

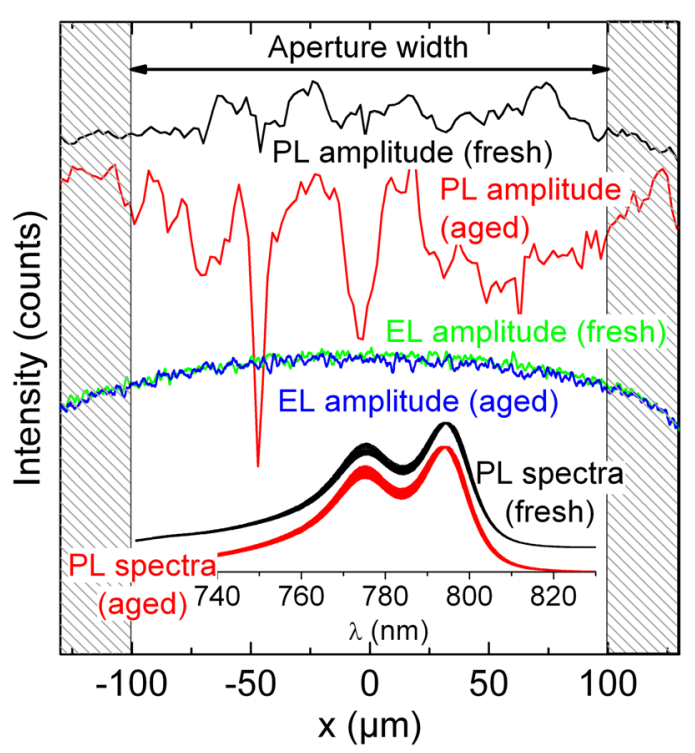

FIG. 2. Evolution of EL and PL taken at the facet of device F before and after $200 \mathrm{~h}$ of accelerated aging under external feedback. The inset shows normalized PL spectra taken along the emitter aperture before and after aging (vertically shifted for clarity, 100 each, $2 \mu \mathrm{m}$ step width). accelerated aging affect the active region of the device. Electroluminescence (EL) mapping and micro-photoluminescence (PL) spectroscopy with excitation through the facet are applied. The inset shows 100 normalized PL spectra taken along the aperture before and after aging. The heavy- and light-hole-related QW emission peaks are at 794 and $775 \mathrm{~nm}$, respectively. After aging, the QW PL spectra are unchanged (also at longer wavelengths, which are not plotted here), while the PL amplitude shows sharp structures such as the drop to about $30 \%$ of the average at $x=-45 \mu \mathrm{m}$, for example. The noticeable dehomogenization of the PL amplitude is in strong contrast to the absence of aging effects on the smooth EL amplitudes, since both EL and PL monitor the QW's spontaneous emission. At this point, however, the unequal carrier generation processes in both types of imaging come into play: while the non-equilibrium carriers, whose recombination produces EL, are generated at the forward biased p-n junction throughout the entire QW-plane, the ones that produce the PL are generated by absorption of the excitation laser photons $\left(\hbar \omega_{\text {exc }}=2.8 \mathrm{eV}\right)$ exclusively in the vicinity of the facet. These high-energy photons are absorbed within $\sim 100 \mathrm{~nm}$ predominantly in the waveguide and cladding layers, rather than in the thin QW. Afterwards, the carriers generated in this way diffuse along the laser axis $(z)$ and drift along the growth direction (y) into the QW, where their recombination generates the PL signal. If there are any laterally spread (point) defects or traps close to the facet in either cladding or waveguide, this will substantially reduce the QW PL, even if there are no defects in the QW. In other words: the "information depth" of EL is larger than that of PL (at least if PL is excited with blue light). Thus, we interpret our findings on PL and EL as a hint that the observed gradual degradation is caused by (obviously laterally inhomogeneously) generated (point) defects or traps close to the facet rather than by defects that are spread within the entire active region along $z$. At this point, however, the question in which layer along $y$-direction, QW, waveguide, or cladding, the defects are indeed located remains open.

In order to identify this location, we opened the devices mechanically by removing the $n$-contact followed by selective etching of the GaAs substrate and buffer layer. ${ }^{7}$ After this, the optical active layer plane (containing QW and waveguide) remains covered only by the $n-\mathrm{Al}_{0.45} \mathrm{Ga}_{0.55} \mathrm{As}$-cladding when looking from the top (Fig. 1(a)). In the following, this plane $(x-z)$ is analyzed by various spectroscopic means.

Figures 3(a)-3(c) shows panchromatic cathodoluminescence (CL, acceleration voltage $25 \mathrm{kV}$ and spatial resolution $\sim 2 \mu \mathrm{m}$ ) images from devices $\mathrm{P}, \mathrm{R}$, and F, respectively, covering the first $\sim 230 \mu \mathrm{m}$ of the $200 \mu \mathrm{m}$ wide emitter stripes (see dotted squares) with the front facet always on top. The substructuring into 20 emitters (vertical stripes) and the increased "overall" darkening of the stripe region with aging are visible. The latter is interpreted as uniform point defect generation related to device operation and not to external feedback. In addition, device $\mathrm{F}$ shows an unexpected peculiarity, highly likely a result of the presence of external feedback: at a depth of about $z=5-20 \mu \mathrm{m}$ (measured from the facet, in sub-figure (c) from top to the bottom) there is a rather blurred area along almost the entire emitter width, which additionally contains small dark spots, see arrow in (c). Subfigure (d) shows a secondary electron (SE) image covering exactly the same area as 

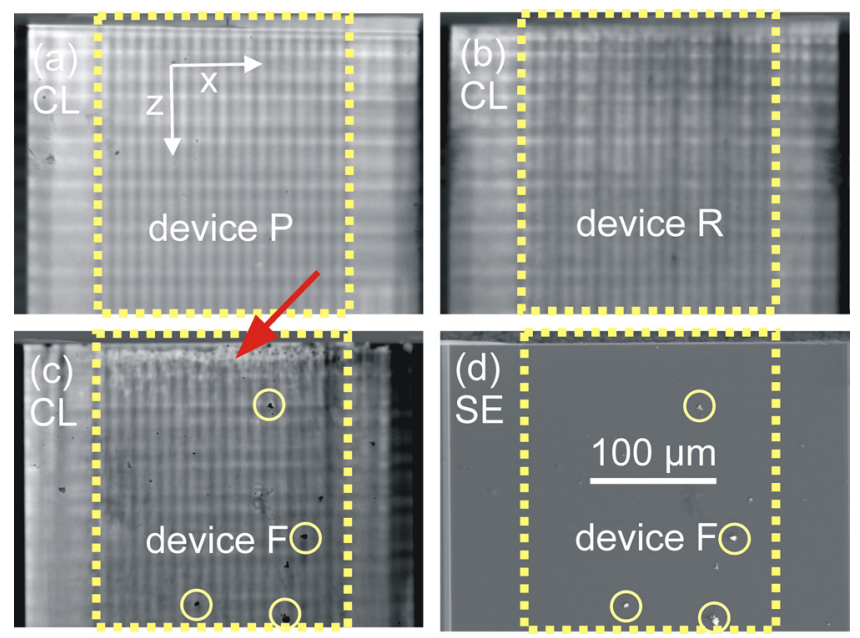

FIG. 3. (a)-(c) Panchromatic CL maps of the close-to-facet regions of device P, R, and F. (d) SE image of the same area of device F. The dotted squares mark the emitter stripe with the facet being located on top. Notice that the stripe extends $1200 \mu \mathrm{m}$ further to the bottom. The arrow in (c) points to a "blurred region" that represents a typical signature of devices aged under external feedback. Circles in (c) and (d) mark examples for etching bumps. These artifacts from preparation appear bright in SE and dark in CL.

shown in (c). It does not show any topographic structures, which potentially could also cause such an effect. Note that the SE image, Fig. 3(d), additionally reveals a number of bright spots, which may be found as dark spots in CL, Fig. 3(c). Some examples are marked by circles in (c) and (d). A correlation is obvious. These are artifacts from preparation (etching bumps) and not directly related to the state of the samples, such as aged or fresh.

Figure 4(a) shows a typical CL spectrum (80 K; acceleration voltage $20 \mathrm{kV}$ ). Note that the electron beam hits the
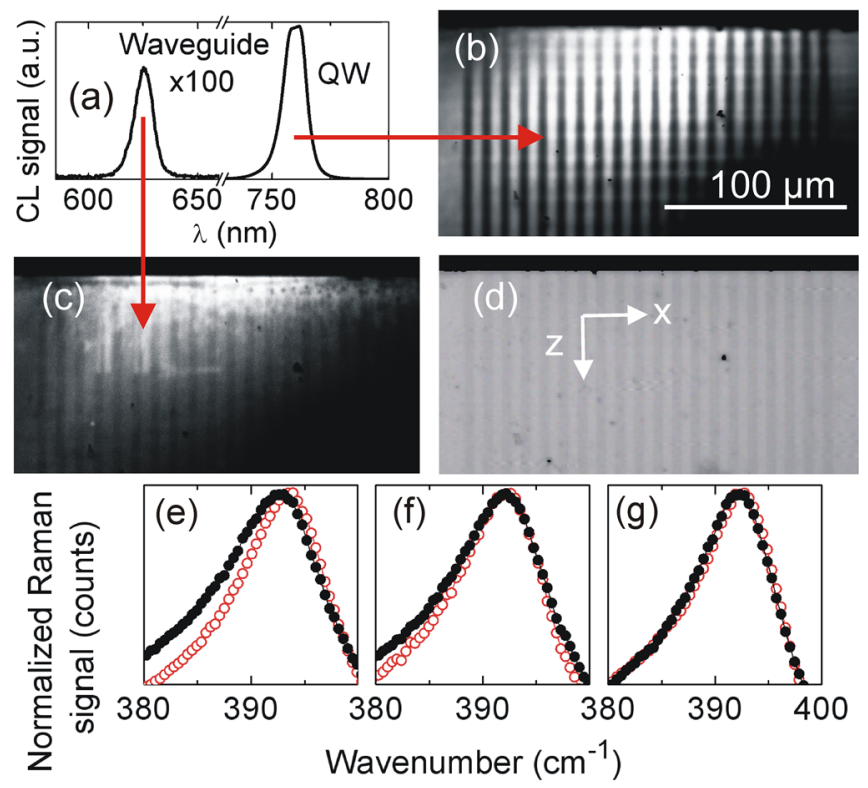

FIG. 4. (a) CL spectrum taken in the center of device F at $80 \mathrm{~K}$. (b) CL map from device $\mathrm{F}$ taken at $760 \mathrm{~nm}$ (QW emission) and $80 \mathrm{~K}$. (c) CL map from device $\mathrm{F}$ taken at $625 \mathrm{~nm}$ (waveguide emission) and $80 \mathrm{~K}$. (d) Ambienttemperature PL map (QW emission) covering the same area as the CL map given in (b). (e)-(g) Raman spectra from the $n$-cladding layers of devices F, $\mathrm{R}$, and $\mathrm{P}$ taken from the close-to-facet regions (full circles) and reference regions within the bulk (open circles). In fact, each spectrum is the sum of 25 spectra scanned along $x$-axis within the respective regions. sample at the $n$-cladding as the topmost surface after removal of the substrate. Carrier drift and diffusion cause the population of QW and waveguide giving rise to the emission lines at 760 and $625 \mathrm{~nm}$, respectively. Subfigures (b) and (c) show the CL maps of QW and waveguide emission, respectively. The data clearly show the peculiarity observed close to the facet in the panchromatic CL from device $\mathrm{F}$, see arrow Fig. 3(c), to be produced by the CL emission of the waveguide layers and not by the QW. This finding is crosschecked by PL microscopy of the same area shown in Fig. 4(d). Here, the infrared excitation (from a halogen lamp; edge filters remove the high-energy parts of the spectrum) passes through the wider-bandgap waveguide and cladding layers and resonantly excites the QW. The result is a homogeneous QW PL pattern in the entire close-to-facet region. This is unambiguous evidence that the observed gradual degradation under external feedback is not, as one could have expected, caused by degradation of QW properties.

Therefore, we proceed with further analysis of the $n$ cladding layer. Figures 4(e)-4(g) shows Raman spectra from devices $\mathrm{F}, \mathrm{R}$, and $\mathrm{P}$ taken at locations close to the facet at about $z=10 \mu \mathrm{m}$ (full circles) and for reference in the interior $z \sim 200 \mu \mathrm{m}$ (open circles). Each is an average of 25 spectra taken at steps of $1 \mu \mathrm{m}$ along $x$. This approach demonstrates that the changes are indeed systematic (averaging does not increase the halfwidth). For device F, the close-to-facet region inspected by the Raman probe is the one marked in Fig. 3(c) by the arrow (starting point of the $25 \mu \mathrm{m}$ scan to the left). Raman spectra from all devices and regions peak in the $392-393 \mathrm{~cm}^{-1}$ range pointing to the AlAs LO phonons in AlAs-rich material providing the main share. ${ }^{8}$ This is consistent with the expectation that after removing the substrate (and buffer), the $\mathrm{Al}_{0.45} \mathrm{Ga}_{0.55} \mathrm{As} n$-cladding material is probed. The asymmetry towards smaller wavenumbers is likely to be caused by AlAs TO phonons. In the given (001)orientation, the selection rules virtually "forbid" TO phonon scattering. Our observation, however, is not surprising for epitaxial systems and is typically assigned to reduced material $^{9}$ or interface quality. ${ }^{8}$ Thus, regarding the parameter "presence of TO phonons" accelerated aging affects device $\mathrm{F}$ most, followed by $\mathrm{R}$, while $\mathrm{P}$ remains, as expected, unaffected.

Exclusively for device $\mathrm{F}$, there is a distinct aginginduced $1 \mathrm{~cm}^{-1}$-shift towards smaller wavenumbers. There are several explanations for such a shift, among them relaxation of compressive built-in-strain, generation of (native) point defects ${ }^{10}$ resulting in tensile strain (widening of the inplane lattice constant), or severe damage due to generation of point defects as common for ion implantation. ${ }^{9}$ Thus, Raman spectroscopy unambiguously proves that the claddings of device $\mathrm{F}$ are severely affected by operation under external feedback, at least on a level that is characterized by point defect creation. Note that analysis of the same area by transmission electron microscopy (TEM) has not revealed any extended defects in $\mathrm{QW}$, waveguide, or the claddings that are obviously modified on a point-defect-level.

The different behaviors of waveguide and QW CL signals in the close-to-facet regions, Figs. 4(b) and 4(c), have another important implication: both CL contributions are generated by recombining carrier pairs also generated in the 
damaged $n$-cladding layer. Before detection, the CL has in both cases to pass again through them. Thus, masking effects are likely on both tracks. At this point, the spatially homogeneous QW CL map (b) clearly proves that the inhomogeneous behavior of the waveguide CL map (c) is not due to any masking effect of the damaged $n$-cladding, but represents the genuine CL behavior of the waveguide.

Another interesting question is the one behind the mechanisms by which external feedback impacts the $n$-cladding and waveguide. Obviously, the feedback increases the absolute load at the facet. An additional potential path is the absorption of the back-reflected $808 \mathrm{~nm}$ laser light by the GaAs substrate on the $n$-side or the cap on the $p$-side. This would lead to additional heating of the front facet region from outside of the active region. If this effect is dominant, our conclusions will be limited to devices with emission wavelengths shorter than $870 \mathrm{~nm}$. Clarification of this issue will be goal of further work.

In summary, we investigated early stages of gradual device degradation that took place under external optical feedback in commercial $808 \mathrm{~nm}$ emitting high-power diode lasers. Accelerated aging tests with $200 \mathrm{~h}$ duration have been carried out. Almost linear gradual power losses of 5.4\% and $2.4 \%$ have been observed with and without external feedback, respectively. Subsequently, these devices, plus unaged reference devices from the same batch, have been analyzed with respect to alterations that are responsible for the gradual power losses observed. PL and EL spectroscopy over the facet revealed that the changes took place underneath the front facet; i.e., not in the bulk, without producing any signatures being perceivable by external inspection. Preparative opening of the devices allowed for the inspection of the dis- tinct layers forming the "active region" of the devices. Clear signatures of (point) defect creation are seen by Raman spectroscopy within the cladding layers. These layers are also the ones containing the highest Al-content. Dehomogenization of the waveguide CL pinpoints alterations also in this layer. At the same time, CL and PL mapping proved the QW was not affected by any perceptible aging effects. This might be the most astonishing result of this study.

Helpful discussions with Stella Elliott and Markus Weyers and expert technical assistance by Sandy Schwirzke-Schaaf, Monika Tischer, and Helen Lawrenz are acknowledged. We are grateful to Dirk Berger and Anna Mogilatenko for TEM investigations. Funding by the European Commission within the project www.BRIGHTER.eu under Contract No. IST-2005035266 is acknowledged.

${ }^{1}$ R. Lang and K. Kobayashi, IEEE J. Quantum Electron. 16, 347-355 (1980).

${ }^{2}$ M. Lobel, P. M. Petersen, and P. M. Johansen, Opt. Lett. 23, 825-827 (1998).

${ }^{3}$ M. Chi, B. Thestrup, and P. M. Petersen, Opt. Lett. 30, 1147-1149 (2005).

${ }^{4}$ J. W. Tomm, M. Ziegler, M. Hempel, and T. Elsaesser, Laser Photon. Rev. 5, 422-441 (2011).

${ }^{5}$ The devices SPL CG81-2S are commercially available.

${ }^{6}$ M. Ziegler, J. W. Tomm, D. Reeber, T. Elsaesser, U. Zeimer, H. E. Larsen, P. M. Petersen, and P. E. Andersen, Appl. Phys. Lett. 94, 191101 (2009).

${ }^{7}$ M. Baeumler, J. L. Weyher, S. Muller, W. Jantz, R. Stibal, G. Herrmann, J. Luft, K. Sporrer, and W. Spath, Defect Recognition and Image Processing in Semiconductors DRIP VII 1997 (1998), Vol. 160, pp. 467-470.

${ }^{8}$ G. Abstreiter, E. Bauser, A. Fischer, and K. Ploog, Appl. Phys. 16, 345352 (1978).

${ }^{9}$ J. W. Tomm, V. Strelchuk, A. Gerhardt, U. Zeimer, M. Zorn, H. Kissel, M. Weyers, and J. Jimenez, J. Appl. Phys. 95, 1122-1126 (2004).

${ }^{10}$ X. Liu, A. Prasad, J. Nishio, E. R. Weber, Z. Liliental-Weber, and W. Walukiewicz, Appl. Phys. Lett. 67, 279-281 (1995). 